\title{
Cellular microparticles in the pathogenesis of pulmonary hypertension
}

\author{
Nicolas Amabile ${ }^{1,2,3,8}$, Christophe Guignabert ${ }^{3,4,5,8}$, David Montani ${ }^{3,4,5,6}$, \\ Yerem Yeghiazarians ${ }^{7}$, Chantal M. Boulanger ${ }^{1,2,8}$ and Marc Humbert $3,4,5,6,8$ \\ Affiliations: \\ ${ }^{1}$ INSERM U970, Paris Cardiovascular Research Center, Paris, \\ ${ }^{2}$ Université Paris Descartes, Sorbonne Paris Cité, UMR-S970, Paris, \\ ${ }^{3}$ Centre Chirurgical Marie Lannelongue, Cardiology Dept and Research Dept, Le Plessis-Robinson, \\ ${ }^{4}$ INSERM U999, LabEx LERMIT, Centre Chirurgical Marie Lannelongue, Le Plessis-Robinson, \\ ${ }^{5}$ Univ Paris-Sud, Faculté de médecine, Le Kremlin-Bicêtre, and \\ ${ }^{6}$ Centre de Référence de l'Hypertension Pulmonaire Sévère, Service de Pneumologie et Réanimation \\ Respiratoire, DHU Thorax Innovation, Assistance Publique Hôpitaux de Paris, Hôpital de Bicêtre, Le Kremlin- \\ Bicêtre, France. \\ ${ }^{7}$ Division of Cardiology, Dept of Medicine, University of California San Francisco, San Francisco, CA, USA. \\ ${ }^{8}$ Authors contributed equally.
}

\section{Correspondence:}

M. Humbert, INSERM U999, Centre Chirurgical Marie Lannelongue, 133 Avenue de la Résistance, 92350 Le Plessis-Robinson, France.

E-mail: marc.humbertabct.aphp.fr

ABSTRACT Pulmonary hypertension $(\mathrm{PH})$ is a fatal disease with no treatment options, characterised by elevated pulmonary vascular resistanzce and secondary right ventricular failure. The aetiology of pulmonary arterial hypertension is multiple and its pathogenesis is complex. Although the exact role of cellular microparticles remains partially understood, there is increasing evidence to suggest an active role for microparticles in $\mathrm{PH}$ pathophysiology. Patients with $\mathrm{PH}$ exhibited higher circulating levels of microparticles compared to control subjects and in vitro or in vivo generated microparticles can induce endothelial dysfunction, interfere with coagulation pathways or modulate inflammatory phenomenon. Whether or not these new conveyors of biological information contribute to the acquisition and/or maintenance of the altered endothelial phenotype is unexplored in $\mathrm{PH}$ and requires further study.

@ERSpublications

Patients with $\mathrm{PH}$ have elevated circulating levels of microparticles which may contribute to disease pathophysiology http://ow.ly/ksnT6

Received: June 022012 | Accepted after revision: Aug 282012 | First published online: Dec 202012

Conflict of interest: Disclosures can be found alongside the online version of this article at www.erj.ersjournals.com Copyright @ERS 2013 


\section{Introduction}

Pulmonary hypertension $(\mathrm{PH})$ is characterised by widespread obstruction and obliteration of pulmonary arterioles, leading to a progressive elevation in pulmonary vascular resistance and subsequent right heart failure and death. The exact mechanisms of pulmonary arterial remodelling that lead to the onset and progression of $\mathrm{PH}$ are still largely unclear. However, many disease-predisposing factors and/or contributing factors have been identified, including inflammation, endothelial cell dysfunction, aberrant vascular wall cell proliferation and mutations in the bone morphogenetic protein-receptor type 2 (Bmpr2) gene [1-3].

Precise and dynamic crosstalk between cells is critical for cellular behaviour, including for cell proliferation, apoptosis, differentiation, migration and survival, and thus crucial for proper tissue organisation and homeostasis; abnormal cellular crosstalk can lead to the development of cancer and PH $[4,5]$. These wellregulated processes to transfer information between cells occur through direct cell-cell contact, various soluble bioactive factors and through cellular microparticles $[6,7]$.

Microparticles are plasma membrane vesicle fragments (between 0.1 and $1 \mu \mathrm{m}$ in size) released from various cell types during activation by agonists or physical or chemical stress, including apoptosis $[6,7]$. Microparticles in body fluids constitute a heterogeneous population, differing in cellular origin (endothelial cells, platelets, leukocytes and erythrocytes), number, size, antigenic composition and functional properties. Their concentration increases specifically and differently during various pathological states, including cardiovascular diseases (table 1) [8]. Indeed, patients with $\mathrm{PH}$ (group 1 and 3 with no associated cardiovascular disease) exhibit higher circulating levels of microparticles compared to control subjects [9-11]. This increase might indicate a potential involvement of microparticles in the disease onset and its progression. This article reviews the evidence supporting potential roles and importance of microparticles in the pathogenesis of $\mathrm{PH}$.

\section{TABLE 1 Microparticle involvement in cardiovascular diseases}

Cardiovascular disease

\section{Cardiovascular risk factors}

Diabetes

Severe systemic hypertension

Metabolic syndrome

Hypertriglyceridemia

Endothelial dysfunction

Acute endothelial dysfunction

Chronic renal failure with endothelial dysfunction

Coronary artery disease with endothelial dysfunction

Diabetes with endothelial dysfunction

Obesity with endothelial dysfunction

Atherosclerosis

Subclinical atherosclerosis

Coronary calcification

Acute coronary syndrome

Stable coronary artery disease

Acute stroke

Cerebrovascular atherosclerosis

Peripheral artery disease

Heart failure and valvular disease

Congestive heart failure

Cyanotic congenital heart disease

Heart transplant rejection

Pulmonary hypertension

Severe aortic stenosis

Atrial fibrillation

Venous thromboembolism

Other vascular diseases

Inflammatory vasculitis

Antiphospholipid syndrome

Pre-eclampsia
Microparticles

TF-MPs, CD8 ${ }^{+}$MPs, $C D 66^{+}$MPs, PMPs, CD51 ${ }^{+}$EMPs, PMPs, $\mathrm{PS}^{+} \mathrm{MPs}$

CD $31^{+}$EMPs, PMPs

CD $31^{+}$EMPs, CD $51^{+}$EMPs

CD31 $1^{+}$EMPs

CD $31^{+}$EMPs, CD144 ${ }^{+}$EMPs, CD62 $e^{+}$EMPs

CD $31^{+}$EMPs, CD $144^{+}$EMPs, PMPs, RBCMPs, $\mathrm{PS}^{+} \mathrm{MPs}$

$\mathrm{CD} 31^{+} / \mathrm{PS}^{+} \mathrm{MPs}$

CD144 ${ }^{+}$EMPs

CD $31^{+} / 42^{-}$EMPs

CD11 $a^{+}$LMPs

CD62e ${ }^{+}$EMPs, PMPs

$\mathrm{PS}^{+} \mathrm{MPs}, \mathrm{CD} 31^{+} \mathrm{MPs}, \mathrm{CD} 146^{+} \mathrm{EMPs}, \mathrm{CD} 31^{+} \mathrm{EMPs}, \mathrm{CD} 51^{+} \mathrm{EMPs}, \mathrm{PMPs}$

CD $31^{+}$EMPs, CD $51^{+}$EMPs

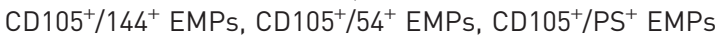

CD62 $\mathrm{e}^{+}$EMPs, CD31 $1^{+}$EMPs

PMPs

CD62e $e^{+}$EMPs, CD $31^{+}$EMPs PMPs

CD62 $\mathrm{e}^{+}$EMPs, $\mathrm{TF}^{+/-}$MPs, FAS ${ }^{-}$MPs

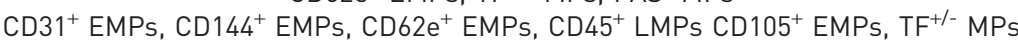

$\mathrm{CD}^{2} \mathrm{e}^{+}$EMPs, CD11 $1^{+} \mathrm{LMPs}$

$\mathrm{PS}^{+} \mathrm{MPs}, \mathrm{PMPs}$

CD $31^{+}$EMPs, CD62 $\mathrm{e}^{+}$EMPs

CD $105^{+}$EMPs, CD62 $e^{+}$EMPs, PMPs, $P^{+}$MPs, PMPs

$\mathrm{CD} 31^{+} / \mathrm{CD} 51^{+} \mathrm{EMPs}$

$\mathrm{CD}^{+}{ }^{+} \mathrm{MPs}, \mathrm{CD}^{+} \mathrm{MPs}, \mathrm{CD} 66^{+} \mathrm{LMPs}^{\mathrm{CD}} 31^{+} \mathrm{EMPs}$

TF: tissue factor; PMPs: platelet microparticles (MPs); EMPs: endothelial microparticles; PS: phosphatidylserine; RBCMPs: red blood cell-derived microparticles; LMPs: leukocyte-derived microparticles. Modified from [8]. 


\section{General considerations on microparticles}

Detectable levels of microparticles of different cellular origin (platelets, red blood cells, leukocytes, endothelial cells) circulate in the plasma of healthy subjects (fig. 1b). Circulating microparticles levels increase in a wide range of cardiovascular diseases, including uncontrolled cardiovascular risk factors, stable and unstable atherosclerotic lesions, heart failure, arrhythmias and inflammatory vascular diseases (table 1) [8]. The presence of microparticles in plasma reflects an active balance between microparticle generation and clearance. Both phagocytic activities present in the liver and the spleen contribute to the removal of microparticles from plasma of healthy animals $[12,13]$. Changes in circulating levels of microparticles might bear important clinical information in healthy subjects and in patients with cardiovascular disorders. Microparticles display various bioactive substances and receptors on their surface and harbour a concentrated set of cytokines, signalling proteins, lipids and nucleic acids. The composition of microparticles, which subtends their biological effect, is a function of the parent cell they stem from, as well as the stimulus used for their generation. Thus, the amount and nature of circulating microparticles influence their beneficial or deleterious biological effects in physiological or pathological conditions. Indeed, various experimental evidence reports significant inter-relationships between circulating microparticles (either generated in vivo or in vitro) and other protagonists of disrupted vascular homeostasis. Microparticles can modulate vascular reactivity (particularly endothelial dependent relaxation), interfere with coagulation pathways, enhance or decrease

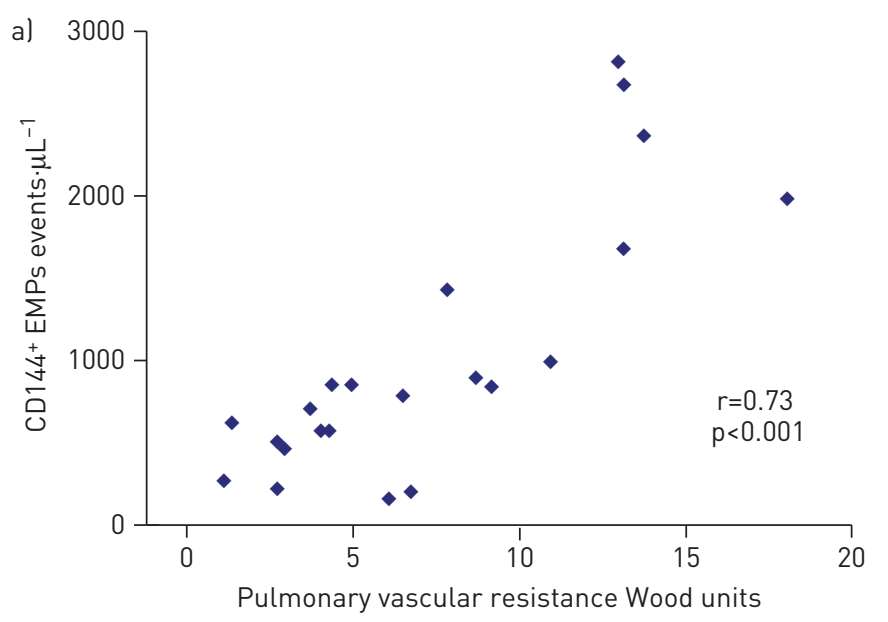

b)
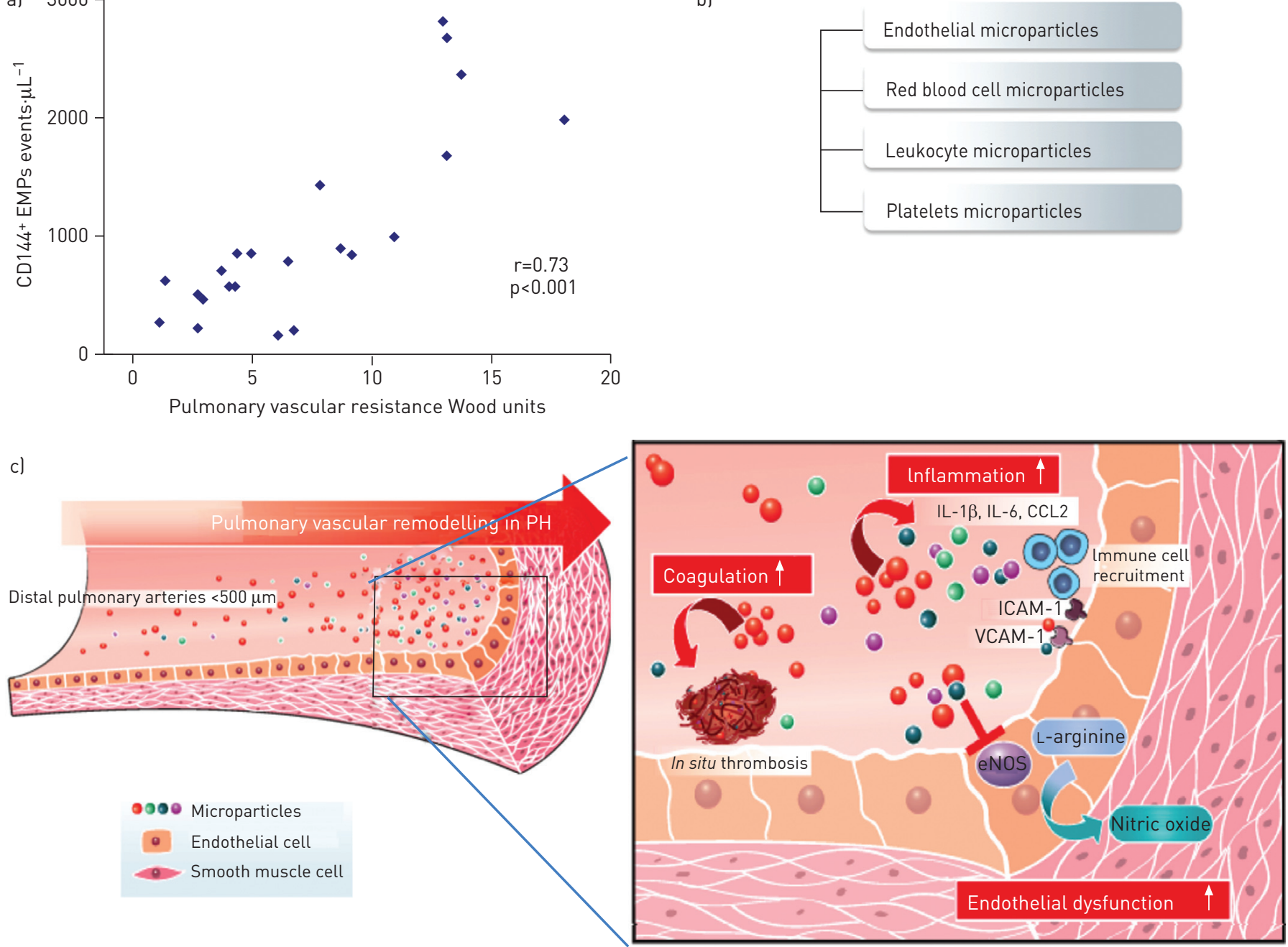

FIGURE 1 a) Relationships between endothelial microparticles (EMPs) and pulmonary vascular resistance in patients with pulmonary hypertension (PH). b) The most important known types of circulating microparticles. c) Proposed model for the role of microparticles in PH pathogenesis. High circulating levels of EMPs in patients with PH (group 1 and 3 with no associated cardiovascular disease) could: 1) contribute, at least in part, to the initiation and/or perpetuation of the endothelial dysfunction and decreased nitric oxide production by pulmonary endothelial cells through an increase in oxidative stress; 2) interfere with coagulation pathways; and/or 3) modulate inflammation through the stimulation of the release of various key cytokines, and/or via induction of intercellular adhesion molecule (ICAM)-1, vascular cell adhesion molecule (VCAM)-1 and E-selectin at the endothelial cell surface. IL: interleukin; CCL2: chemokine ligand 2; eNOS: endothelial nitric oxide synthase. 
inflammatory phenomenon and influence neoangiogenesis processes. A large part of our knowledge regarding microparticles is based on data issued from the research on athero-thrombotic disease [14]. However, recent data shed light on their role as vectors of cellular crosstalk with implications in the pathogenesis of nonatherosclerotic cardiovascular conditions, including pulmonary vascular diseases (table 1).

\section{Circulating microparticles as regulators of endothelial dysfunction}

Endothelial dysfunction is a pivotal element in the development and progression of $\mathrm{PH}$, irrespective of disease origin [15]. The dysfunctional endothelium displays, to varying degrees, an imbalanced production of several mediators (vasoconstrictors versus vasodilators; activators versus inhibitors of smooth muscle cell growth; pro-inflammatory versus anti-inflammatory signals; prothrombotic versus antithrombotic substances) leading towards an excess of vasoconstriction, smooth muscle hyperplasia and pulmonary vascular remodelling [1-3]. In addition, disturbances of the normal balance between endothelial cell proliferation and endothelial cell apoptosis have been reported in idiopathic pulmonary arterial hypertension $(\mathrm{PAH})$ [16, 17]. Indeed, accumulating evidence supports the concept that increased endothelial apoptosis at the early stage and decreased endothelial apoptosis at later stages of the disease could contribute to PAH [18]. Consistent with this hypothesis, we have recently reported that primary pulmonary endothelial cells generated from PAH lung specimens exhibit various intrinsic abnormalities and present a modified pro-proliferative, apoptotic-resistant phenotype [4, 17, 19]. Although we have shown that increased activity of the FGF2 autocrine loop is among the mechanisms needed to acquire this altered endothelial phenotype in $\mathrm{PAH}$ [17], the exact nature of pulmonary endothelial cell modification during $\mathrm{PAH}$ and the balance between apoptotic and anti-apoptotic phenotypes remain only partially understood. In addition, alterations of endothelial cell monolayer integrity in mice, such as a selective disruption of the endothelial peroxisome proliferator-activated receptor- $\gamma$ or of the endothelial BMPR2 signalling, are sufficient to predispose to $\mathrm{PH}[20,21]$.

Different groups have reported increased concentrations of different subsets of circulating endothelial microparticles (EMPs) in PH patients compared to control subjects [9-11], supporting the presence of an increased structural damage with enhanced cellular activation and/or apoptosis. Indeed, the levels of platelet endothelial cell adhesion molecule $\left(\mathrm{PECAM}^{+}\right)$and vascular endothelial (VE)-cadherin ${ }^{+}$EMPs were also correlated with several haemodynamics pulmonary vascular remodelling parameters (fig. 1) [10] and inflammation biomarkers [11]. The aetiology of the disease also appears to influence the microparticles levels: AmABile et al. [10] reported that PAH patients had higher levels of EMPs compared with chronic pulmonary disease-related PH subjects, whereas DiEHL et al. [11] observed higher levels of EMPs bearing Eselectin in thromboembolic $\mathrm{PH}$ compared with non-thromboembolic $\mathrm{PH}$ subjects.

The origin of these circulating microparticles (systemic versus pulmonary endothelium) is still a matter of debate. Although a trans-pulmonary gradient of $\mathrm{CD} 105^{+}$(endoglin) EMPs has been observed, suggesting a local release of microparticles within the pulmonary vascular bed, no significant change in $\mathrm{CD} 31^{+}$(PECAM), $\mathrm{CD}_{144}{ }^{+}$(VE-cadherin) and CD62 $\mathrm{e}^{+}$(E-selectin) EMP levels was observed through the pulmonary vasculature $[9,10]$. These findings, observed in small samples of patients with various $\mathrm{PH}$ origins, could reflect the heterogeneity of the endothelial changes among patients with pulmonary vascular disease, depending on the underlying aetiology or the lesion development stage (early versus late stage). These findings might support the notion that some specific EMP subpopulations could be released by either systemic or pulmonary endothelium. Finally, it cannot be excluded that a lack of sensitivity of the measurement methods for detection of mild variations in concentrations of microparticles could also explain these results.

The circulating microparticles are essential vectors of biological information and are involved in cellular communication through a paracrine or endocrine action [22]. Cellular effects of microparticles are directly influenced by the various antigens harboured at their surface, which is a function of the cellular origin and the stimulus involved in the vesiculation process [8]. In vitro or in vivo generated microparticles can induce endothelial dysfunction, interfere with coagulation pathways or modulate inflammatory phenomenon [8]. Accordingly, Tual-Chalot et al. [23] reported that circulating microparticles isolated from hypoxiainduced PH rats could affect the vascular tone. They showed that these microparticles, mainly of platelet and erythrocyte origin, impaired the endothelium-dependent vaso-relaxation in pulmonary arteries ex vivo and decreased the nitric oxide (NO) production by pulmonary endothelial cells through an increase in oxidative stress [23]. The authors observed comparable results in aorta and pulmonary arteries [23]. Taken together, all these experimental findings support the notion that circulating microparticles could contribute to both initiation and/or amplification of the pulmonary endothelial dysfunction during $\mathrm{PH}$ and encourage further studies of the precise pathogenic mechanism underlying this phenomenon. Moreover, we cannot exclude that the generation of microparticles and their migration to the systemic circulation could mediate a crosstalk between the pulmonary and systemic endothelium and, therefore, explain the peripheral endothelial dysfunction observed during $\mathrm{PH}[21,24,25]$. 
Finally, measurement of circulating EMPs might also represent a potential new prognostic tool in patients with PH. Preliminary data reported that baseline values of circulating $\mathrm{CD} 62 \mathrm{e}^{+}$microparticles, measured before initiation of any specific therapy, predicted occurrence of adverse outcome (death and readmission for right heart failure) in a small cohort of patients with mixed aetiologies of $\mathrm{PH}$ [26]. Whereas these results were not observed for other EMP subsets (CD144 ${ }^{+}$and $\mathrm{CD} 31^{+}$EMPs) and should be confirmed in a larger population sample, they are in line with data reported in coronary artery disease [27], heart failure [28] and chronic end-stage renal failure [29], and thus confirm their potential role as a biomarker for outcome prediction in patients with cardiovascular diseases [30].

\section{Circulating microparticles as regulators of inflammation}

It is now well established that inflammation plays an important role in PH in humans: histological studies of PAH lung specimens demonstrate a prominent perivascular infiltration composed of macrophages, dendritic cells, T- and B-lymphocytes, and mast cells; circulating levels of certain cytokines and chemokines are elevated including interleukin (IL)-1 $\beta$, IL-6, IL-8, CX3CL1, CCL2, CCL5 and tumour necrosis factor- $\alpha$ serum levels, and these may correlate with a worse clinical outcome. Evidence supporting a role for inflammation in the progression of the disease has been reviewed recently by PRICE et al. [31]. However, the initial process triggering inflammation onset remains unknown.

Cellular microparticles are key factors in the inflammatory process because they contribute to the endothelial production of various pro-inflammatory cytokines and chemokines. Microparticles have been shown to stimulate the release of IL-1 $\beta$, IL-6, CCL2, and induce the expression of intercellular adhesion molecule (ICAM)-1, vascular cell adhesion molecule (VCAM)-1 and E-selectin [32-35]. In addition, microparticles provide a preferential substrate for non-pancreatic secretory phospholipase A2 and thus a source of lysophosphatic acid, a potent pro-inflammatory mediator and platelet agonist [36]. Furthermore, a recent study has shown that microparticles from septic shock patients increase the expression of endothelial and inducible NO synthase, cyclooxygenase -2 and nuclear factor- $\kappa \mathrm{B}$ in the heart and lung [37]. In addition, DENSMORE et al. [38] have demonstrated that endothelium-derived microparticle sequestration in the lung is capable of initiating inflammatory cascades at pathophysiologically relevant concentrations [38, 39]. Altogether, these findings support the notion that circulating microparticles could actively contribute to the initiation and/or amplification of pulmonary vascular inflammation in the pathogenesis of $\mathrm{PH}$.

\section{Procoagulant properties of microparticles}

Thrombosis is a key mechanism in onset and progression of cardiovascular diseases, including venous thromboembolic disease, atherosclerosis and $\mathrm{PH}$. Chronic thromboembolic $\mathrm{PH}$ results from the obstruction of the pulmonary arteries by clot fragments that have migrated from deep venous thrombosis [40]. However, PAH pathological specimens often display thrombotic lesions in the absence of clinical or pathological evidence of pulmonary embolism [41], suggesting an in situ clotting phenomenon [42]. Furthermore, $\mathrm{PH}$ is associated with a hyper-coagulable phenotype [43] that includes vascular upregulation of tissue factor [44] and an increase in circulating levels of Von Willebrand factor or fibrinopeptide A [45].

Microparticles are involved in thrombus generation by different mechanisms. Microparticles can provide the anionic phospholipid surface necessary for the assembly of clotting enzyme complexes (VIIIa and Xa) promoting thrombin generation. In some cases, microparticles also directly expose tissue factor, the main cellular initiator of blood coagulation, at their surface. Furthermore, microparticles can also harbour other functional membrane or cytoplasmic effectors (such as selectins, GPIIb-IIIa, GPIb, Von Willebrand factor, arachidonic acid, thromboxane A2) that can promote prothrombotic responses [22]. Therefore, microparticles can promote thrombin generation in vitro, but also favour thrombus propagation in vivo [46] or transfer their procoagulant potential to target cells. The thrombogenic potential of microparticles has been demonstrated for several subpopulations, including platelet- [47], endothelial- [48, 49] or leukocyte-derived microparticles [50].

Most of our knowledge regarding microparticles' procoagulant activity derives from data observed in atherosclerosis, cardiac surgery and conditions associated with cardiomyocyte inflammatory stimulation, such as cardiac ischaemia.

An increase in circulating global procoagulants as well as tissue factor $\left(\mathrm{TF}^{+}\right)$microparticle levels has been reported in patients with PAH [9]. Furthermore, the levels of $\mathrm{TF}^{+}$microparticles and Von Willebrand factor were correlated. Interestingly, the highest levels of $\mathrm{TF}^{+}$-microparticles were observed in patients with functional class III-IV and/or the weakest 6-min test performance, suggesting a potential correlation between microparticles and functional status. Comparable results were observed in patients with recurrent deep venous thrombosis, a potential trigger for acute pulmonary embolism development [51]. 
The precise molecular mechanisms governing these biological effects of microparticles are still under investigation, but several elements support the notion that procoagulant microparticles might interfere with the coagulation process in $\mathrm{PH}$ patients and promote the disease development in different ways. High levels of procoagulant circulating microparticles could favour recurrent deep venous thrombosis [52], which will in turn create silent pulmonary embolism and ultimately lead to post-thromboembolic $\mathrm{PH}[40,53]$. Conversely, the plexiform lesions and the dysfunctional endothelium could release procoagulant microparticles, which could lead to propagation of the thrombotic process among the pulmonary vessels. The resulting local thrombosis could worsen the microvascular obstruction and thus increase pulmonary artery pressure. This hypothesis is supported by the results from BAKOUBOULA et al. [9], who observed an increase in the concentration of the global number of procoagulant microparticles between the jugular vein and the wedged pulmonary artery, suggesting a local release of procoagulant microvesicles. However, the authors reported no significant transpulmonary gradient of $\mathrm{TF}^{+}$microparticles, which might be explained by the small size of the group $(n=20)$ and the heterogeneous aetiologies of $\mathrm{PH}$ [9]. Moreover, proteomics analysis in experimental models of post-thromboembolic $\mathrm{PH}$ has shown that this condition was associated with increased expression of coagulation proteins carried by circulating microparticles [54].

\section{Potential implications of microparticles in PH pathophysiology and remaining questions}

In the light of the data mentioned above, the involvement of microparticles in $\mathrm{PH}$ pathogenesis seems to be, at least, plausible. Experimental and clinical data suggest multiple roles of microparticles in the progression of the disease and one might hypothesise a possible global scheme (fig. 1c). Procoagulant microparticles can enhance local and systemic thrombogenicity leading to pulmonary artery clot. Moreover, pro-inflammatory microparticles could also increase endothelial inflammation, local cytokine and growth factor release. Furthermore, microparticles might also favour endothelial cell proliferation. Finally, circulating microparticles can enhance endothelial dysfunction, decrease NO release and deteriorate the vasodilation/vasoconstriction humoral balance. Altogether, these effects can support pulmonary vessel remodelling and vascular obstruction and should encourage further in vivo and in vitro studies to identify their precise pathogenic mechanisms. In addition, a very recent study has reported that epoprostenol could inhibit human platelet-leukocyte mixed conjugate and platelet microparticle formation in whole blood, demonstrating that treatments might interfere with microparticles and some of their biological roles [55]. Since our knowledge of microparticles properties in vivo might also be influenced by the current methods used for their isolation and their measurement, there is a need to establish a consensus regarding the standard centrifugation method to prepare poor platelets plasma from whole blood samples in order to determine microparticle phenotypes and levels. To date, the appropriate method for microparticles measurement is still a matter of debate [56]. Flow cytometry methods, which have been widely used during the past 20 years, need small sample volumes and allow complete phenotypical analysis. However, flow cytometry doesn't analyse microparticles function and is limited by its poor sensitivity, resulting from the biophysical and optical properties of the laser interaction with small vesicles $(<500 \mu \mathrm{m})$. The "capture" and other purely biological methods inform on microparticles function but don't provide any direct quantification. Dynamic light scattering, atomic force microscopy, automated flow through single-particle optical microscopy or impedance based flow cytometry represent promising methods for the future, allowing more accurate microparticle quantification, but these methods are not currently adapted for clinical research as they are limited by their cost or their availability.

In addition to these potential pathogenic roles of microparticles in $\mathrm{PH}$, microparticles are well-known modulators of different stages of angiogenesis. These particular effects of microparticles depend on the stimulation and activation status of the cell from which they originate and also on their concentration $[57,58]$. Indeed, microparticles from platelets display pro-angiogenic properties by promoting capillary-like structures and pro-angiogenic factor production [59]. By contrast, lymphocyte- and endothelial-derived microparticles exhibit pro- or anti-angiogenic properties depending on the stimuli used for their production [57, 60-63]. These additional properties of microparticles reinforce the idea that they could be potential key actors in $\mathrm{PH}$ pathogenesis in the early-, middle- and/or late-stage of the disease.

Whether or not these new conveyors of biological information contribute to the acquisition and/or maintenance of the altered endothelial phenotype is not fully explored in $\mathrm{PH}$ and requires further study. Thus, several questions remain regarding their nature and true importance in this disease. What are the compositions of microparticles in $\mathrm{PH}$ and what are their functions? What causes increased amounts of circulating microparticles in PH? Are they beneficial or deleterious in PH? Are they useful for disease recurrence, prognosis and monitoring of therapeutic efficacy? By which potential mechanism(s) do microparticles initiate and propagate $\mathrm{PH}$ ? 


\section{Conclusion}

Patients with PH exhibit higher circulating levels of microparticles compared to control subjects and it is unclear whether these new conveyors of biological information contribute to the acquisition and/or maintenance of the altered endothelial phenotype or are just a consequence of the disease progression. However, there is strong accumulating evidence that microparticles are key protagonists of the disease and they could serve as a worthy biomarker for prognosis stratification and therapeutic efficiency monitoring. Therefore, a better understanding of their importance in the disease is essential and efforts should be made in the future to improve our knowledge by further exploring cellular aspects as well as clinical implications of microparticles in pulmonary hypertension.

\section{References}

1 Humbert M, Morrell NW, Archer SL, et al. Cellular and molecular pathobiology of pulmonary arterial hypertension. J Am Coll Cardiol 2004; 43: Suppl. 12, 13S-24S.

2 Morrell NW, Adnot S, Archer SL, et al. Cellular and molecular basis of pulmonary arterial hypertension. J Am Coll Cardiol 2009; 54: Suppl. 1, S20-S31.

3 Rabinovitch M. Molecular pathogenesis of pulmonary arterial hypertension. J Clin Invest 2008; 118: 2372-2379.

4 Eddahibi S, Guignabert C, Barlier-Mur AM, et al. Cross talk between endothelial and smooth muscle cells in pulmonary hypertension: critical role for serotonin-induced smooth muscle hyperplasia. Circulation 2006; 113: $1857-1864$.

$5 \mathrm{Xu}$ R, Mao JH. Gene transcriptional networks integrate microenvironmental signals in human breast cancer. Integr Biol (Camb) 2011; 3: 368-374.

6 Diamant M, Tushuizen ME, Sturk A, et al. Cellular microparticles: new players in the field of vascular disease? Eur J Clin Invest 2004; 34: 392-401.

Freyssinet JM. Cellular microparticles: what are they bad or good for? J Thromb Haemost 2003; 1: 1655-1662. Amabile N, Rautou PE, Tedgui A, et al. Microparticles: key protagonists in cardiovascular disorders. Semin Thromb Hemost 2010; 36: 907-916.

9 Bakouboula B, Morel O, Faure A, et al. Procoagulant membrane microparticles correlate with the severity of pulmonary arterial hypertension. Am I Respir Crit Care Med 2008; 177: 536-543.

10 Amabile N, Heiss C, Real WM, et al. Circulating endothelial microparticle levels predict hemodynamic severity of pulmonary hypertension. Am J Respir Crit Care Med 2008; 177: 1268-1275.

11 Diehl P, Aleker M, Helbing T, et al. Increased platelet, leukocyte and endothelial microparticles predict enhanced coagulation and vascular inflammation in pulmonary hypertension. J Thromb Thrombolysis 2011; 31: 173-179.

12 Dasgupta SK, Abdel-Monem H, Niravath P, et al. Lactadherin and clearance of platelet-derived microvesicles. Blood 2009; 113: 1332-1339.

13 Willekens FL, Werre JM, Kruijt JK, et al. Liver Kupffer cells rapidly remove red blood cell-derived vesicles from the circulation by scavenger receptors. Blood 2005; 105: 2141-2145.

14 Rautou PE, Vion AC, Amabile N, et al. Microparticles, vascular function, and atherothrombosis. Circ Res 2011; 109: 593-606.

15 Budhiraja R, Tuder RM, Hassoun PM. Endothelial dysfunction in pulmonary hypertension. Circulation 2004; 109: 159-165.

16 Masri FA, Xu W, Comhair SA, et al. Hyperproliferative apoptosis-resistant endothelial cells in idiopathic pulmonary arterial hypertension. Am J Physiol Lung Cell Mol Physiol 2007; 293: L548-L554.

17 Tu L, Dewachter L, Gore B, et al. Autocrine fibroblast growth factor-2 signaling contributes to altered endothelial phenotype in pulmonary hypertension. Am J Respir Cell Mol Biol 2011; 45: 311-322.

18 Sakao S, Taraseviciene-Stewart L, Lee JD, et al. Initial apoptosis is followed by increased proliferation of apoptosisresistant endothelial cells. FASEB J 2005; 19: 1178-1180.

$19 \mathrm{Tu}$ L, De Man FS, Girerd B, et al. A critical role for p130Cas in the progression of pulmonary hypertension in humans and rodents. Am J Respir Crit Care Med 2012; 186: 666-676.

20 Guignabert C, Alvira CM, Alastalo TP, et al. Tie2-mediated loss of peroxisome proliferator-activated $\gamma$ in mice causes PDGF receptor- $\beta$-dependent pulmonary arterial muscularization. Am J Physiol Lung Cell Mol Physiol 2009; 297: L1082-L1090.

21 Hong KH, Lee YJ, Lee E, et al. Genetic ablation of the BMPR2 gene in pulmonary endothelium is sufficient to predispose to pulmonary arterial hypertension. Circulation 2008; 118: 722-730.

22 Morel O, Toti F, Hugel B, et al. Procoagulant microparticles: disrupting the vascular homeostasis equation? Arterioscler Thromb Vasc Biol 2006; 26: 2594-2604.

23 Tual-Chalot S, Guibert C, Muller B, et al. Circulating microparticles from pulmonary hypertensive rats induce endothelial dysfunction. Am J Respir Crit Care Med 2010; 182: 261-268.

24 Friedman D, Szmuszkovicz J, Rabai M, et al. Systemic endothelial dysfunction in children with idiopathic pulmonary arterial hypertension correlates with disease severity. J Heart Lung Transplant 2012; 31: 642-647.

25 Peled N, Bendayan D, Shitrit D, et al. Peripheral endothelial dysfunction in patients with pulmonary arterial hypertension. Respir Med 2008; 102: 1791-1796

26 Amabile N, Heiss C, Chang V, et al. Increased CD62e(+) endothelial microparticle levels predict poor outcome in pulmonary hypertension patients. J Heart Lung Transplant 2009; 28: 1081-1086.

27 Nozaki T, Sugiyama S, Koga $\mathrm{H}$, et al. Significance of a multiple biomarkers strategy including endothelial dysfunction to improve risk stratification for cardiovascular events in patients at high risk for coronary heart disease. J Am Coll Cardiol 2009; 54: 601-608.

28 Nozaki T, Sugiyama S, Sugamura K, et al. Prognostic value of endothelial microparticles in patients with heart failure. Eur J Heart Fail 2010; 12: 1223-1228.

29 Amabile N, Guerin AP, Tedgui A, et al. Predictive value of circulating endothelial microparticles for cardiovascular mortality in end-stage renal failure: a pilot study. Nephrol Dial Transplant 2012; 27: 1873-1880. 
30 Amabile N, Boulanger CM. Circulating microparticle levels in patients with coronary artery disease: a new indicator of vulnerability? Eur Heart J 2011; 32: 1958-1960.

31 Price LC, Wort SJ, Perros F, et al. Inflammation in pulmonary arterial hypertension. Chest 2012; 141: 210-221.

32 MacKenzie A, Wilson HL, Kiss-Toth E, et al. Rapid secretion of interleukin-1 $\beta$ by microvesicle shedding. Immunity 2001; 15: 825-835.

33 Nomura S, Tandon NN, Nakamura T, et al. High-shear-stress-induced activation of platelets and microparticles enhances expression of cell adhesion molecules in THP-1 and endothelial cells. Atherosclerosis 2001; 158: 277-287.

34 Rautou PE, Leroyer AS, Ramkhelawon B, et al. Microparticles from human atherosclerotic plaques promote endothelial ICAM-1-dependent monocyte adhesion and transendothelial migration. Circ Res 2011; 108: 335-343.

35 Vince RV, McNaughton LR, Taylor L, et al. Release of VCAM-1 associated endothelial microparticles following simulated SCUBA dives. Eur J Appl Physiol 2009; 105: 507-513.

36 Fourcade O, Simon MF, Viode C, et al. Secretory phospholipase A2 generates the novel lipid mediator lysophosphatidic acid in membrane microvesicles shed from activated cells. Cell 1995; 80: 919-927.

37 Mastronardi ML, Mostefai HA, Meziani F, et al. Circulating microparticles from septic shock patients exert differential tissue expression of enzymes related to inflammation and oxidative stress. Crit Care Med 2011; 39: $1739-1748$.

38 Densmore JC, Signorino PR, Ou J, et al. Endothelium-derived microparticles induce endothelial dysfunction and acute lung injury. Shock 2006; 26: 464-471.

39 Buesing KL, Densmore JC, Kaul S, et al. Endothelial microparticles induce inflammation in acute lung injury. J Surg Res 2011; 166: 32-39.

40 Pengo V, Lensing AWA, Prins $\mathrm{MH}$, et al. Incidence of chronic thromboembolic pulmonary hypertension after pulmonary embolism. $N$ Engl J Med 2004; 350: 2257-2264.

41 Pietra GG, Edwards WD, Kay JM, et al. Histopathology of primary pulmonary hypertension. A qualitative and quantitative study of pulmonary blood vessels from 58 patients in the National Heart, Lung, and Blood Institute, Primary Pulmonary Hypertension Registry. Circulation 1989; 80: 1198-1206.

42 Bjornsson J, Edwards WD. Primary pulmonary hypertension: a histopathologic study of 80 cases. Mayo Clinic Proc 1985; 60: 16-25.

43 Schermuly RT, Ghofrani HA, Wilkins MR, et al. Mechanisms of disease: pulmonary arterial hypertension. Nat Rev Cardiol 2011; 8: 443-455.

44 White RJ, Meoli DF, Swarthout RF, et al. Plexiform-like lesions and increased tissue factor expression in a rat model of severe pulmonary arterial hypertension. Am J Physiol Lung Cell Mol Physiol 2007; 293: L583-L590.

45 Johnson SR, Granton JT, Mehta S. Thrombotic arteriopathy and anticoagulation in pulmonary hypertension. Chest 2006; 130: 545-552.

46 Furie B, Furie BC. Mechanisms of thrombus formation. N Engl J Med 2008; 359: 938-949.

47 Muller I, Klocke A, Alex M, et al. Intravascular tissue factor initiates coagulation via circulating microvesicles and platelets. FASEB J 2003; 17: 476-478.

48 Abid Hussein MN, Boing AN, Biro E, et al. Phospholipid composition of in vitro endothelial microparticles and their in vivo thrombogenic properties. Thromb Res 2008; 121: 865-871.

49 Combes V, Simon AC, Grau GE, et al. In vitro generation of endothelial microparticles and possible prothrombotic activity in patients with lupus anticoagulant. J Clin Invest 1999; 104: 93-102.

50 Satta N, Toti F, Feugeas O, et al. Monocyte vesiculation is a possible mechanism for dissemination of membraneassociated procoagulant activities and adhesion molecules after stimulation by lipopolysaccharide. J Immunol 1994; 153: 3245-3255.

51 Ye R, Ye C, Huang Y, et al. Circulating tissue factor positive microparticles in patients with acute recurrent deep venous thrombosis. Thromb Res 2012; 130: 253-258

52 Bucciarelli P, Martinelli I, Artoni A, et al. Circulating microparticles and risk of venous thromboembolism. Thromb Res 2012; 129: 591-597.

53 Kearon C. Natural history of venous thromboembolism. Circulation 2003; 107: Suppl. 1, I22-I30.

54 Watts JA, Lee YY, Gellar MA, et al. Proteomics of microparticles after experimental pulmonary embolism. Thromb Res 2012; 130: 122-128.

55 Tamburrelli C, Crescente M, Izzi B, et al. Epoprostenol inhibits human platelet-leukocyte mixed conjugate and platelet microparticle formation in whole blood. Thromb Res 2011; 128: 446-451.

56 Freyssinet JM, Toti F. Membrane microparticle determination: at least seeing what's being sized. J Thromb Haemost 2010; 8: 311-314.

57 Yang C, Mwaikambo BR, Zhu T, et al. Lymphocytic microparticles inhibit angiogenesis by stimulating oxidative stress and negatively regulating VEGF-induced pathways. Am J Physiol Regul Integr Comp Physiol 2008; 294: R467-R476.

58 Lacroix R, Sabatier F, Mialhe A, et al. Activation of plasminogen into plasmin at the surface of endothelial microparticles: a mechanism that modulates angiogenic properties of endothelial progenitor cells in vitro. Blood 2007; 110: 2432-2439.

59 Brill A, Dashevsky O, Rivo J, et al. Platelet-derived microparticles induce angiogenesis and stimulate post-ischemic revascularization. Cardiovasc Res 2005; 67: 30-38.

60 Mezentsev A, Merks RM, O’Riordan E, et al. Endothelial microparticles affect angiogenesis in vitro: role of oxidative stress. Am J Physiol Heart Circ Physiol 2005; 289: H1106-H1114.

61 Leroyer AS, Ebrahimian TG, Cochain C, et al. Microparticles from ischemic muscle promotes postnatal vasculogenesis. Circulation 2009; 119: 2808-2817.

62 Ou ZJ, Chang FJ, Luo D, et al. Endothelium-derived microparticles inhibit angiogenesis in the heart and enhance the inhibitory effects of hypercholesterolemia on angiogenesis. Am J Physiol Endocrinol Metab 2011; 300: E661-E668.

63 Reich N, Beyer C, Gelse K, et al. Microparticles stimulate angiogenesis by inducing ELR+ CXC-chemokines in synovial fibroblasts. J Cell Mol Med 2011; 15: 756-762. 